\title{
Kac-Moody Groups, Topology of the Dirac Determinant Bundle, and Fermionization
}

\author{
Jouko Mickelsson *
}

Research Institute for Theoretical Physics, University of Helsinki, SF-00260 Helsinki, Finland

\begin{abstract}
The relation between Kac-Moody groups and algebras and the determinant line bundle of the massless Dirac operator in two dimensions is clarified. Analogous objects are studied in four space-time dimensions and a generalization of Witten's fermionization mechanism is presented in terms of the topology of the Dirac determinant bundle.
\end{abstract}

\section{Introduction}

Loop groups and their central extensions in relation to Kac-Moody algebras have recently attracted great interest. The theory of loop groups is important among others in understanding completely integrable non-linear systems of KdV type [1], constructing solutions for self-dual Yang-Mills equations [2], string models in particle physics [3], and anomaly problems in quantum field theory [4].

The purpose of the present paper is twofold. First, we shall study the non-trivial $U(1)$ bundle $P$ on the space $\Omega G$ of loops in a Lie group. This bundle has a Lie group structure such that the corresponding Lie algebra is a Kac-Moody algebra based on the Lie algebra of $G$. The structure of the group $P$ has been earlier studied in detail in $[1,5,6]$. The physically important realization in [1] is that the sections of an associated line bundle $E$ form in a natural way a Hilbert space which has a canonical realization as the Fock space for fermions in $1+1$ dimensions. There is a second construction of $P$ directly in terms of local charts in $\Omega G$, transition functions and local two-cocycles [6]. However, we feel that the subject is important enough to deserve a third construction; our construction is very simple and it makes clear the relation of sections of $E$ to sections of the determinant line bundle of the $(1+1)$-dimensional Dirac operator.

The second main point in this paper is to clarify the topological and geometrical structure of the Dirac determinant line bundle in $3+1$ dimensions. Particular attention is paid to the case $G=S U(2)$; this case is important for

* Permanent address: Department of Mathematics, University of Jyväskylä, Seminaarinkatu 15, SF-40100 Jyväskylä, Finland 
understanding the Fermi-Bose relation in field theory. Some time ago Witten [7] showed that solitons in the Wess-Zumino model (with an anomaly term in the Lagrangian) have fermionic properties. The basic idea is as follows [8]. Let $f_{0}: S^{3} \rightarrow S U(2)$ represent a static soliton with a winding number

$$
n=\pi_{3}(f)=\frac{1}{24 \pi^{2}} \int \operatorname{tr}\left(d f f^{-1}\right)^{3} .
$$

If one rotates slowly the soliton as time $-\infty \leqq t \leqq+\infty, f(\mathbf{x}, t)=U(t) f_{0}(\mathbf{x}) U(t)^{-1}$, by the matrix $U(t)$ around a fixed coordinate axes, then the contribution to the Lagrangian from the anomaly term is $\delta \mathscr{L}(f)=i \pi n$ and it gives rise to a factor $(-1)^{n}=\exp -\delta \mathscr{L}(f)$ for the quantum mechanical action $S=\exp -\mathscr{L}$. Thus $f$ behaves like a fermion if $n$ is odd.

We shall extend Witten's $\sigma$-model result to Yang-Mills theory using the topology of the field space. In the case $G=S U(2)$ each connected component of the space $\mathscr{A}_{4} / \mathscr{G}_{4}$ of gauge orbits has the first fundamental group equal to $\mathbb{Z}_{2}$ and moreover $\pi_{2}\left(\mathscr{A}_{4} / \mathscr{G}_{4}\right)=\mathbb{Z}_{2}$. From this fact follows that the determinant bundle on $\mathscr{A}_{4} / \mathscr{G}_{4}$ [which is an associated bundle to a $U(1)$ principal bundle] is reducible to a $\mathbb{Z}_{2}$ bundle. The determinant bundle Det ${ }_{4}$ has a natural connection which reflects this reduction phenomenon. Namely, if one takes a non-contractible loop $\gamma$ in $\mathscr{A}_{4} / \mathscr{G}_{4}$, then the parallel transport along $\gamma$ in $\operatorname{Det}_{4}$ is given by a multiplication by the number $(-1)^{n}$, where $n$ is again the soliton number indexing the different connected components of $\mathscr{A}_{4} / \mathscr{G}_{4}$. The space $\Gamma$ of sections in Det ${ }_{4}$ can be thus divided into a sum of subspaces $\Gamma_{+}$and $\Gamma_{-}$corresponding to the behaviour of sections under parallel transport around closed loops (multiplication by \pm 1 ). On the other hand, a non-contractible loop corresponds to a rotation of the fields around a fixed coordinate axes. Thus $\Gamma_{-}$represents fermions and $\Gamma_{+}$bosons.

In the effective action formulation of a coupled Dirac and Yang-Mills field (the Dirac field is integrated away) the Schrödinger wave functions of the system are complex-valued functions $\psi$ on $\mathscr{A}_{4}$ which satisfy the gauge anomaly condition [17], i.e. they are sections of the bundle $\operatorname{Det}_{4}$, [9]. From the topological decomposition $\Gamma=\Gamma_{+} \oplus \Gamma_{-}$follows the important confirmation for the usefulness of the effective action method: half of the wave functions indeed describe fermions and the second half bosons.

\section{The Dirac Determinant Bundle in Two Dimensions}

Let $G$ be a connected, simply connected semi-simple Lie group [for example, $S U(N)$ for $N \geqq 2]$. Any principal $G$ bundle over the compactified space-time $M=S^{2}$ is topologically trivial and any $G$ connection on $S^{2}$ can be given by a globally defined $\mathfrak{g}$-valued $\left(\mathfrak{g}\right.$ is the Lie algebra of $G$ ) one-form on $S^{2}$. Let $\mathscr{A}$ be the linear space of all these one-forms. Let $m \in S^{2}$ be a fixed point (the north pole) and $\mathscr{G}$ the group of smooth maps $f: S^{2} \rightarrow G$ with $f(m)=1$.

We can think of $\mathscr{A}$ also as the space of vector potentials $A$ on the unit disc with appropriate boundary values on $S^{1}=\partial D$ [such that $A$ is a pullback of a smooth potential on $S^{2}$ under the mapping $D \ni(r, \varphi)$ $\mapsto(-\cos \pi r, \sin \pi r \cos \varphi, \sin \pi r \sin \varphi) \in S^{2}, 0 \leqq r \leqq 1 ;$ the circle $r=1$ corresponds 
to the point $\left.m \in S^{2}\right]$. The gauge group $\mathscr{G}$ consists then of maps $f: D \rightarrow G$ with $f(x)=1$ for all $x \in S^{1}$. For each $A \in \mathscr{A}$ there exists a unique $V_{A}: D \rightarrow G$ such that the radial component of $\tilde{A}=V_{A}^{-1} A V_{A}+V_{A}^{-1} d V_{A}$ vanishes and $V_{A}(p)=1$, where $p \in \partial D$ is some fixed point. Namely, since the bundle is trivial, we can consider a parallel transport along a given path as an element of $G$. Let $U_{A}(x)$ be the parallel transport along a straight line from the origin of $D$ to the point $x$. Then $V_{A}(x)=U_{A}(x) U_{A}^{-1}(p)$ has the required properties. Any gauge transformation which preserves the condition $A_{r}=0$ must be a function of the angle $\varphi$ only; the only smooth functions in $D$ of this type are all constants. The condition $V_{A}(p)=1$ fixes the constant uniquely. It is clear that if $A^{\prime}$ is in the same gauge class in $\mathscr{A} / \mathscr{G}$ as $A$, then $V_{A}$ and $V_{A^{\prime}}$ are equal on the boundary $S^{1}$; thus we have a well-defined map $\mathscr{A} / \mathscr{G} \rightarrow \Omega G$ with $\Omega G:=\left\{f: S^{1} \rightarrow G \mid f(p)=1\right\}$. This map is a homotopy equivalence $\mathscr{A} / \mathscr{G} \cong \Omega G,[10]$. Let $D G:=\{f: D \rightarrow G \mid f(p)=1\}$. Then $D G$ is a principal bundle over the base space $\Omega G$, the projection $D G \rightarrow \Omega G$ being the restriction map $\left.f \mapsto f\right|_{S^{1}}$. Each fiber is isomorphic to the space of maps $f: D \rightarrow G$ which are equal to 1 on the boundary $S^{1}$; such $f$ can be thought of as a map $f: S^{2} \rightarrow G$. Thus the fiber can be identified as the group $\mathscr{G}$. The correspondence $A \mapsto V_{A}$ is a $\mathscr{G}$-bundle map from $\mathscr{A}$ into $D G$. The right action of $\mathscr{G}$ on $D G$ is just given by pointwise right multiplication.

The bundle $\mathscr{A} \rightarrow \mathscr{A} / \mathscr{G}$ has a natural connection [10]. This connection is in fact a pull-back of a connection on the homotopically equivalent bundle $D G \rightarrow \Omega G$. We shall now describe in some detail the latter connection. For each $f \in D G$ the tangent space $T_{f} D G$ can be thought of as the space of maps $X: D \rightarrow \mathfrak{g}$ with $X(p)=0$. Define

$$
(X, Y)=\int_{D}\left\langle\partial^{k}(\operatorname{Ad}(f) X), \partial_{k}(\operatorname{Ad}(f) Y)\right\rangle d^{2} x .
$$

Here $\langle\cdot, \cdot\rangle$ is an invariant bilinear form in $\mathfrak{g}$, and the standard Euclidean metric is used in $D$. The vertical subspace $V_{f} \subset T_{f} D G$ for the projection $D G \rightarrow \Omega G$ consists of elements $X: D \rightarrow \mathfrak{g}$ which are zero on the boundary. We define the horizontal subspace $H_{f}$ as the orthogonal complement of $V_{f}$ with respect to the form $(\cdot, \cdot)$. From (1) it follows that

$$
H_{f}=\left\{X \in T_{f} D G \mid \Delta(\operatorname{Ad}(f) X)=0\right\} .
$$

If $X \in T_{f}$ is arbitrary, there exists a unique harmonic function $Z: D \rightarrow \mathfrak{g}$ such that $\left.Z\right|_{S^{1}}=\left.\operatorname{Ad}(f) X\right|_{S^{1}}$. The horizontal projection of $X$ is now given by $h X=\operatorname{Ad}\left(f^{-1}\right) Z$. The distribution $f \mapsto H_{f}$ is equivarinat with respect to the right action of $\mathscr{G}$ on $D G$, therefore it defines a connection.

We shall construct the principal $U(1)$ bundle $P$ on $\Omega G$ which was mentioned in the introduction. Consider the set $D G \times U(1)$ with the equivalence relation

$$
(f, \lambda) \sim(f g, \lambda \exp 2 \pi i \omega(f, g)),
$$

where $g \in \mathscr{G}$ and $\omega$ is the real valued function defined by

$$
\begin{gathered}
\omega(f, g)=\frac{\theta^{2}}{16 \pi^{2}} \int_{D}\left\langle f^{-1} d f, d g g^{-1}\right\rangle+C(g), \\
C(g)=\frac{-\theta^{2}}{48 \pi^{2}} \int_{D_{3}} \varepsilon^{\alpha \beta \gamma}\left\langle g^{-1} \partial_{\alpha} g, \frac{1}{2}\left[g^{-1} \partial_{\beta} g, g^{-1} \partial_{\gamma} g\right]\right\rangle .
\end{gathered}
$$


Here $g: S^{2} \rightarrow G$ has been extended in an arbitrary way to $g: D_{3} \rightarrow G$, $D_{3}=\left\{x \in \mathbb{R}^{3} \mid\|x\| \leqq 1\right\}$. (Remember that $g: D \rightarrow G$ with $\left.g\right|_{S^{1}}=1$ gives a map $g: S^{2} \rightarrow G$.) The number $\theta^{2}$ is (length) ${ }^{2}$ of the longest root of the Lie algebra $g$; in particular, if we take $\langle A, B\rangle=\operatorname{tr} A B$ in the defining representation of $S U(N)$, then $\theta^{2}=2,[16]$.

For any two extensions $g_{1}, g_{2}$ one has $C\left(g_{1}\right)-C\left(g_{2}\right) \in \mathbb{Z}$. Thus exp2 $2 \pi i \omega(f, g)$ is well-defined. We define $P=(D G \times U(1)) / \sim$.

The bundle $P$ has a group structure. The product of two equivalence classes in $D G \times U(1)$ is

$$
\begin{gathered}
{[(f, \lambda)] \cdot\left[\left(f^{\prime}, \lambda^{\prime}\right)\right]=\left[\left(f f^{\prime}, \lambda \lambda^{\prime} \exp 2 \pi i \gamma\left(f, f^{\prime}\right)\right)\right],} \\
\gamma\left(f, f^{\prime}\right)=\frac{\theta^{2}}{16 \pi^{2}} \int_{D}\left\langle f^{-1} d f, d f^{\prime} f^{\prime-1}\right\rangle .
\end{gathered}
$$

One has to prove that the class on the right-hand side of (3) is independent of the particular representatives on the left-hand side. Using Stokes' theorem, we get first

$$
C\left(f^{-1} g f\right)=C(g)+\gamma\left(f^{-1} g, f\right)+\gamma\left(f^{-1}, g\right) .
$$

Here we have extended $f: D \rightarrow G$ to a cone $T$ such that $D$ is the base of $T ; g$ is extended similarly with the constraint $g=1$ on the conical surface $S$. Surface integrals over $S$ vanish since

$$
\gamma\left(f^{-1} g, f\right)=\gamma\left(f^{-1}, f\right) \equiv 0 \quad \text { on } S .
$$

Let $(\tilde{f}, \tilde{\lambda}) \sim(f, \lambda)$, that is $\tilde{f}=f g$ and $\tilde{\lambda}=\lambda \exp 2 \pi i \omega(f, g)$ for some $g$ with $\left.g\right|_{S^{1}}=1$. Now $\left(\widetilde{f} f^{\prime}, \tilde{\lambda} \lambda^{\prime} \exp 2 \pi i \gamma\left(\tilde{f}, f^{\prime}\right)\right)$ should represent the same class as $\left(f f^{\prime}, \lambda \lambda^{\prime} \exp 2 \pi i \gamma\left(f, f^{\prime}\right)\right)$. This is equivalent to

$$
\gamma\left(f, f^{\prime}\right)+\omega\left(f f^{\prime}, f^{\prime-1} g f^{\prime}\right) \equiv \omega(f, g)+\gamma\left(f g, f^{\prime}\right) \bmod \mathbb{Z} .
$$

Using (5) we see that Eq. (6) follows from

$$
\gamma\left(f, f^{\prime}\right)+\gamma\left(f f^{\prime}, f^{\prime-1} g f^{\prime}\right)+\gamma\left(f^{\prime-1} g, f^{\prime}\right)+\gamma\left(f^{\prime-1}, g\right) \equiv \gamma(f, g)+\gamma\left(f g, f^{\prime}\right),
$$

which in turn can be verified by a straightforward computation. One has to show also that the product depends only on the class represented by the second factor $\left(f^{\prime}, \lambda^{\prime}\right)$; this is the easier part of the proof and is left to the reader. Note that

$$
\gamma\left(f_{1}, f_{2}\right)+\gamma\left(f_{1} f_{2}, f_{3}\right)=\gamma\left(f_{2}, f_{3}\right)+\gamma\left(f_{1}, f_{2} f_{3}\right),
$$

from which follows the associativity of the product (3). [Equation (7) means that $\exp 2 \pi i \gamma$ is a $U(1)$ valued two-cocycle in $D G$.]

The group cocycle $\gamma$ defines a Lie algebra cocycle for $\operatorname{Map}(D, \mathfrak{g})$,

$$
\frac{1}{2 \pi} c(X, Y)=\left.2 \frac{d^{2}}{d t d s} \gamma\left(e^{t X}, e^{s Y}\right)\right|_{t=s=0}=\frac{\theta^{2}}{8 \pi^{2}} \int_{D}\langle d X, d Y\rangle=\frac{\theta^{2}}{8 \pi^{2}} \int_{S^{1}}\langle X, d Y\rangle .
$$

Since $c(X, Y)$ depends only on the boundary values of $X$ and $Y$, it defines in fact a two-cocycle for the Lie algebra $\operatorname{Map}\left(S^{1}, \mathfrak{g}\right)$; the central extension of $\operatorname{Map}\left(S^{1}, \mathfrak{g}\right)$ defined by the cocycle $c$ is the Kac-Moody algebra based on $\mathrm{g}$. The group $P$ is not the Kac-Moody group in the usual sense, since we are considering here only based loops $(f(p)=1)$; letting $f(p)$ be arbitrary, one gets the Kac-Moody group $\hat{P}$ 
associated to $G ; P \cong \hat{P} / G$. Because of the non-trivial topology of the bundle $P$, the group cocycle on $\Omega G$ corresponding to $c$ cannot be globally defined; one has to divide the space $\Omega G$ into an infinite number of coordinate patches and define the cocycle separately on each patch; see [6] for details.

We define a connection in the bundle $P$. Consider first $D G \times U(1)$ as a principal $\mathscr{G} \times U(1)$ bundle over $\Omega G$ with the action

$$
(f, \lambda) \mapsto(f g, \lambda \mu \exp 2 \pi i \omega(f, g))
$$

of $(g, \mu) \in \mathscr{G} \times U(1)$ on $D G \times U(1)$. We define a connection in $D G \times U(1)$ by declaring the horizontal subspace $H_{(f, \lambda)}$ at $(f, \lambda)$ to consist of pairs $(X, a) \in T_{f} D G$ $\times \mathbb{R}$ with

$$
a=\frac{\theta^{2}}{8 \pi} \int_{D}\left\langle f^{-1} d f, d X\right\rangle .
$$

The horizontal subspace $H_{[(f, \lambda)]}$ for $P$ at $[(f, \lambda)]$ is then the image of $H_{(f, \lambda)}$ under the canonical projection $D G \times U(1) \rightarrow(D G \times U(1)) / \mathscr{G}$. The space $H_{[(f, \lambda)]}$ depends only on the class $[(f, \lambda)]$ and not on the representative $(f, \lambda)$. Namely, if $(X, a)$ is a tangent vector at $(f, \lambda)$ then the image of $(X, a)$ under the canonical projection is the same as the image of the vector $\left(g^{-1} X g, a+\frac{\theta^{2}}{8 \pi} \int\left\langle d X+\left[f^{-1} d t, X\right], d g g^{-1}\right\rangle\right)$ at the equivalent point $(f g, \lambda \exp 2 \pi i \omega(f, g))$; the latter vector is horizontal iff the former is. Since the action of $\mathscr{G}$ in $D G \times U(1)$ commutes with the $U(1)$ action, the distribution $H_{[(f, \lambda)]}$ in $P$ is equivariant with respect to the $U(1)$ action. So $H$ is indeed a connection in the principal bundle $P$. The connection one-form in the total space of the bundle $D G \times U(1)$ is particularly simple: at $(f, \lambda)$ the value of this form for the tangent vector $(X, a)$ is

$$
-a+\frac{\theta^{2}}{8 \pi} \int_{D}\left\langle f^{-1} d f, d X\right\rangle .
$$

Let us compute the (real-valued) vector potential $\alpha$ on $\Omega G$ corresponding to the connection $H$ on $P$. First, we have to fix a gauge (a local section of $P) f \mapsto[(\widetilde{f}, 1)]$, where $f \in \Omega G$ and $\tilde{f}$ is an extension of $f$ to $D$. For example, near the unit element in $G$, we can write $f=\exp X$ and $\tilde{f}=\exp \widetilde{X}, \widetilde{X}$ being the unique harmonic extension of $X$ to $D$. When $X \in T_{f} \Omega G$,

$$
\frac{1}{2 \pi} \alpha_{f}(X)=\frac{\theta^{2}}{16 \pi^{2}} \int_{D}\left\langle\tilde{f}^{-1} d \tilde{f}, d(\tilde{X})\right\rangle .
$$

The curvature computed from $\alpha$ is

$$
d \alpha_{f}(X, Y)=\frac{\theta^{2}}{4 \pi} \int_{S^{1}}\langle X, d Y\rangle,
$$

which is just the central term $c(X, Y)$ of the Kac-Moody algebra.

The natural action of $U(1)$ on complex numbers defines a complex line bundle $E$ over $\Omega G$. The elements of $E$ are equivalence classes of pairs $(f, \lambda) \in D G \times \mathbb{C}$ with respect to the equivalence relation $(f, \lambda) \sim(f g, \lambda \exp 2 \pi i \omega(f, g)), g \in \mathscr{G}$. The space $\Gamma(E)$ of sections of $E$ consists therefore of functions $\psi: D G \rightarrow \mathbb{C}$ such that

$$
\psi(f g)=\psi(f) \cdot \exp 2 \pi i \omega(f, g) .
$$


On the other hand, the sections of the determinant bundle Det for the left-handed Dirac operator $\not+i A P_{+}\left(P_{+}\right.$is the projection on the left-handed components of the Dirac field in $1+1$ space-time dimensions) are complex-valued functions $\psi$ on $\mathscr{A}$ such that

$$
\psi\left(g^{-1} A g+g^{-1} d g\right)=\psi(A) \exp 2 \pi i \bar{\omega}(A, g)
$$

for $A \in \mathscr{A}, g \in \mathscr{G}$; here

$$
\bar{\omega}(A, g)=\frac{\theta^{2}}{16 \pi^{2}} \int_{D}\left\langle A, d g g^{-1}\right\rangle+C(g) .
$$

The functional $\bar{\omega}(A, g)$ is known as the chiral anomaly in physics literature. The bundle Det is the pull-back of $E$ under the mapping $A \mapsto V_{A}^{-1}$. Namely, let $\psi$ be any section of $E$, i.e. a function $\psi: D G \rightarrow \mathbb{C}$ satisfying (11). Define $\tilde{\psi}: \mathscr{A} \rightarrow \mathbb{C}$, $\tilde{\psi}(A)=\psi\left(V_{A}^{-1}\right)$. Then

$$
\tilde{\psi}\left(g^{-1} A g+g^{-1} d g\right)=\tilde{\psi}(A) \exp 2 \pi i \omega\left(V_{A}^{-1}, g\right) .
$$

On the other hand,

$$
\bar{\omega}(A, g)=\omega\left(V_{A}^{-1}, g\right)+F\left(g^{-1} A g+g^{-1} d g\right)-F(A),
$$

where

$$
F(A)=-\int\left\langle A, d V_{A} V_{A}^{-1}\right\rangle .
$$

The Eq. (15) is an expression for the fact that the cocycles $\omega\left(V_{A}^{-1}, g\right)$ and $\bar{\omega}(A, g)$ represent the same cohomology class; here $\omega\left(V_{A}^{-1}, g\right)$ is considered as a function of $A$ and $g$. If one defines $\varphi(A)=\tilde{\psi}(A) \cdot \exp 2 \pi i F(A)$, then $\varphi$ satisfies Eq. (12).

Representations of Kac-Moody algebras can be used to produce sections of the bundle $E$ (and therefore also of the bundle Det). The highest weight representations of the Kac-Moody algebra $\hat{\mathrm{g}}$ associated to $G$ can be exponentiated to representations of the group $\hat{P},[11]$. Given a representation $T$ of $\hat{P}$ in a Hilbert space $\mathscr{H}$ such that elements $e^{-i \varphi}$ in the centre are represented by the complex number $e^{-i \varphi}$, one can associate to each pair of vectors $v_{0}, v \in \mathscr{H}$ a complex function

$$
\psi(f)=\left(v, T([(f, 1)]) v_{0}\right), \quad f \in D G ;
$$

$(\cdot, \cdot)$ is the inner product in $\mathscr{H}$. It is clear from the definition of $\hat{P}$ that $\psi$ satisfies (11). Thus $\psi$ is a section of $E$. In fact, it is shown in [1] that all holomorphic sections of the dual bundle $E^{*}$ can be obtained in this way using the basic representation of $\hat{P}$ in the fermionic Fock space. By this construction one gets a correspondence between sections of the determinant bundle and the fermion state vectors.

\section{The Determinant Bundle in Four Dimensions and the Fermi-Bose Relation}

In this section $G=S U(N), N \geqq 2$. Let $\mathscr{A}_{4}$ be the space of connection forms on $S^{4}$, with values in the Lie algebra $\mathfrak{g}$, and let $\mathscr{G}_{4}$ be the group of pointed gauge transformations, $f(m)=1$ for all $f \in \mathscr{G}_{4}$ for some fixed $p \in S^{4}$. We denote by $D_{4}$ the unit disc in $\mathbb{R}^{4}, \partial D_{4}=S^{3}$, and $D_{4} G=\left\{f: D_{4} \rightarrow G \mid f(p)=1\right\}$, where $p \in \partial D_{4}$ is fixed. Since $\pi_{3} G=\mathbb{Z}$, the space $\Omega_{3} G=\left\{f: S^{3} \rightarrow G \mid f(p)=1\right\}$ is disconnected, and the connected components $\Omega_{3}^{(n)} G$ are labelled by the soliton number $n \in \mathbb{Z}$. Each connected component (indexed by the instanton number) of the bundle $\mathscr{A}_{4} \rightarrow \mathscr{A}_{4} / \mathscr{G}_{4}$ is homotopically equivalent to $D_{4} G \rightarrow \Omega_{3}^{(0)} G$. The group $\mathscr{G}_{4}$ can be identified as the subgroup $\left\{f: D_{4} \rightarrow G \mid f(x)=1 \forall x \in \partial D_{4}\right\} \subset D_{4} G$. In the following 
we shall restrict to the zero soliton sector, and for brevity we denote $\Omega_{3}=\Omega_{3}^{(0)} G$; at the end of this section we shall make some remarks about the $n$-soliton sector.

A connection and the curvature can be defined in the bundle $\mathscr{A}_{4} \rightarrow \mathscr{A}_{4} / \mathscr{G}_{4}$ or in $D_{4} G \rightarrow \Omega_{3} G$ as in the two dimensional case, [10]. However, we shall go over directly to the determinant bundle $\operatorname{Det}_{4}$ on $\mathscr{A}_{4} / \mathscr{G}_{4}$. The regularized determinant of the Dirac operator $\not+i A P_{+}$in four dimensions is a complex-valued function $\psi$ on $\mathscr{A}_{4}$ such that [9]

$$
\begin{aligned}
& \psi\left(g^{-1} A g+g^{-1} d g\right)=\psi(A) \exp 2 \pi i \bar{\omega}(A, g), \\
& \bar{\omega}(A, g)= \frac{i}{24 \pi^{3}} \int \operatorname{tr}\left[-d g g^{-1}\left(\frac{1}{2} A d A+\frac{1}{2} d A A+\frac{1}{2} A^{3}\right)\right. \\
&\left.+\frac{1}{4}\left(d g g^{-1} A\right)^{2}+\frac{1}{2}\left(d g g^{-1}\right)^{3} A\right]+C_{5}(g) \\
&= \bar{\omega}_{0}(A, g)+C_{5}(g),
\end{aligned}
$$

where the integral is over the disc $D_{4}$ (we have removed one point, say $m$, from $S^{4}$ and projected the rest to $\left.D_{4}\right), g \in \mathscr{G}_{4}$ and $C_{5}$ is an integral of a topological density when $N \geqq 3$,

$$
C_{5}(g)=\frac{i}{240 \pi^{3}} \int_{D_{5}} \operatorname{tr}\left(d g g^{-1}\right)^{5} .
$$

Here $g: S^{4} \rightarrow S U(N)$ has been extended in an arbitrary way to a map $g: D_{5} \rightarrow S U(N)$. If $N=2$ the density in (18) is identically zero, since a five-form on a $3 D$ manifold vanishes. In the following we shall consider the case $N \geqq 3$, and we shall return to $N=2$ at the end of this section.

The anomaly $\bar{\omega}$ satisfies the Wess-Zumino consistency condition, or put differently, it is a 1-cocycle of the group $\mathscr{G}_{4},[17]$.

The functions $\psi$ satisfying (17) can be considered as sections of a bundle Det $_{4}$ on $\mathscr{A}_{4} / \mathscr{G}_{4}$. By definition, elements of $D_{\text {et }}$ are equivalence classes in $\mathscr{A}_{4} \times \mathbb{C}$ with respect to the equivalence relation

for $g \in \mathscr{G}_{4}$.

$$
(A, \lambda) \sim\left(g^{-1} A g+g^{-1} d g, \lambda \exp 2 \pi i \bar{\omega}(A, g)\right)
$$

There exists an analogous object to the bundle $P$. By definition, the bundle $P_{3}$ on $\Omega_{3} G$ consists of equivalence classes $[(f, \lambda)]$ in $D_{4} G \times U(1)$ with respect to the relation

$$
(f, \lambda) \sim(f g, \lambda \exp 2 \pi i \omega(f, g)), \quad \omega(f, g)=\bar{\omega}\left(f^{-1} d f, g\right)
$$

for $g \in \mathscr{G}_{4}$. However, the bundle $P_{3}$ does not have any natural group structure like in the case of $P$. In order to understand the reason for this, let us first define a twococycle $\gamma\left(A ; g_{1}, g_{2}\right)$ for the group $D_{4} G$,

$$
\begin{aligned}
\gamma\left(A ; g_{1}, g_{2}\right):= & \left(\delta \bar{\omega}_{0}\right)\left(A ; g_{1}, g_{2}\right) \\
= & \bar{\omega}_{0}\left(A, g_{1}\right)+\bar{\omega}_{0}\left(g_{1}^{-1} A g_{1}+g_{1}^{-1} d g_{1}, g_{2}\right)-\bar{\omega}_{0}\left(A, g_{1} g_{2}\right) \\
= & -\frac{i}{24 \pi^{3}} \int_{S^{3}} \operatorname{tr}\left[-\frac{1}{2}\left(d g_{2} g_{2}^{-1}\right)\left(g_{1}^{-1} d g_{1}\right)\left(g_{1}^{-1} A g_{1}\right)\right. \\
& \left.+\frac{1}{2}\left(d g_{2} g_{2}^{-1}\right)\left(g_{1}^{-1} A g_{1}\right)\left(g_{1}^{-1} d g_{1}\right)\right]+\frac{i}{24 \pi^{3}} \int_{D_{4}} \operatorname{tr}\left[\frac{1}{2}\left(d g_{2} g_{2}^{-1}\right)\left(g_{1}^{-1} d g_{1}\right)^{3}\right. \\
& \left.+\frac{1}{4}\left(d g_{2} g_{2}^{-1} g_{1}^{-1} d g_{1}\right)^{2}+\frac{1}{2}\left(d g_{2} g_{2}^{-1}\right)^{3}\left(g_{1}^{-1} d g_{1}\right)\right]
\end{aligned}
$$


The square of the coboundary operator $\delta$ is zero and therefore $\gamma$ is indeed a twococycle; the difference compared with the two dimensional case is, that $\gamma$ depends on the vector potential $A$. For this reason the proper group extension of $D_{4} G$ is not just by $U(1)$ but by the abelian group $\operatorname{Map}\left(\mathscr{A}_{3}, U(1)\right)$ (pointwise multiplication, $\mathscr{A}_{3}$ is the space of $\mathrm{g}$-valued vector potentials in $S^{3}$ ),

$$
(f, \lambda) \cdot\left(f^{\prime}, \lambda^{\prime}\right)=\left(f f^{\prime}, \lambda \lambda_{f}^{\prime} \exp 2 \pi i \gamma\left(A ; f, f^{\prime}\right)\right),
$$

where $\lambda_{f}(A):=\lambda\left(f^{-1} A f+f^{-1} d f\right)$. Here the gauge transformation for $A \in \mathscr{A}_{3}$ is defined by the restriction of $f$ to $S^{3}=\partial D_{4}$. In the $2 D$ case we defined the group $P$ by first defining the extension of $D G$ by $U(1)$ and then dividing by the equivalence relation " $"$. Analogously, we can define here the equivalence

$$
(f, \lambda) \sim(f g, \lambda \exp 2 \pi i \omega(f, g))
$$

for $g \in \mathscr{G}_{4}$. The set of equivalence classes $\left(D_{4} G \times \operatorname{Map}\left(\mathscr{A}_{3}, U(1)\right)\right) / \sim=Q_{3}$ is a principal bundle on $\Omega_{3} G$ with the structure group $\operatorname{Map}\left(\mathscr{A}_{3}, U(1)\right)$ and it has a group structure inherited from $D_{4} G \times \operatorname{Map}\left(\mathscr{A}_{3}, U(1)\right)$ (see below). One can also consider $Q_{3}$ as an associated bundle to $P_{3}$ via the natural action of $U(1)$ in the space $\operatorname{Map}\left(\mathscr{A}_{3}, U(1)\right)$.

We have to show that the product (22) is well-defined in the space of equivalence classes. We shall again leave to the reader the easier part of the proof: namely, replacing $\left(f^{\prime}, \lambda^{\prime}\right)$ by an equivalent pair on the left-hand side does not change the class on the right-hand side. Let instead replace $(f, \lambda)$ by $(\tilde{f}, \tilde{\lambda})$ with $\tilde{f}=f g$ and $\tilde{\lambda}=\lambda \exp 2 \pi i \omega(f, g)$. The relation $(f, \lambda)\left(f^{\prime}, \lambda\right) \sim(\tilde{f}, \tilde{\lambda})\left(f^{\prime}, \lambda^{\prime}\right)$ is equivalent to the equation

$$
\omega(f, g)+\gamma\left(A ; f g, f^{\prime}\right) \equiv \omega\left(f f^{\prime}, f^{\prime-1} g f^{\prime}\right)+\gamma\left(A ; f, f^{\prime}\right) \bmod \mathbb{Z} .
$$

We have taken into account that $\lambda_{f}^{\prime}=\lambda_{\tilde{f}}^{\prime}$, since $f=\tilde{f}$ on $S^{3}$. From (17), (20), and (21) follows that

$$
\omega(f, g)=\gamma(0 ; f, g)+C_{5}(g) .
$$

Using Stokes' theorem as in the two-dimensional case,

$$
C_{5}\left(f^{-1} g f\right)=C_{5}(g)+\gamma\left(0 ; f^{-1} g, f\right)+\gamma\left(0 ; f^{-1}, g\right) .
$$

Taking account that the terms involving the potential $A$ are equal on both sides of (24) $\left(g=1\right.$ on the boundary $\left.S^{3}\right)$ and combining (25), and (26) we can conclude that (24) is equivalent with

$$
\begin{aligned}
& \gamma(0 ; f, g)+\gamma\left(0 ; f g, f^{\prime}\right) \\
& \quad \equiv \gamma\left(0 ; f^{\prime-1} g, f^{\prime}\right)+\gamma\left(0 ; f f^{\prime}, f^{\prime-1} g f^{\prime}\right)+\gamma\left(0 ; f, f^{\prime}\right)+\gamma\left(0 ; f^{\prime-1}, g\right),
\end{aligned}
$$

modulo integers. This equation can be verified by a simple substitution from (21).

The associativity of the product (22) follows again from the 2-cocycle property of $\gamma ; \gamma$ is trivially a 2-cocycle since it is (in $\left.D_{4}\right)$ the coboundary of $\bar{\omega}_{0}(A, f)$.

The Lie algebra two-cocycle $c_{3}$ in $\operatorname{Map}\left(D_{4}, \mathfrak{g}\right)$ corresponding to the group cocycle $\gamma$ can be obtained from the infinitesimal form [18]

$$
\alpha(A ; X)=\left.2 \pi \frac{d}{d t} \bar{\omega}\left(A, e^{t X}\right)\right|_{t=0}=\frac{i}{12 \pi^{2}} \int \operatorname{tr}\left(\frac{1}{2} A d A+\frac{1}{2} d A A+\frac{1}{2} A^{3}\right) d X
$$


of the gauge anomaly,

$$
\begin{aligned}
c_{3}(A ; X, Y) & =(\delta \alpha)(A ; X, Y)=\delta_{X} \alpha(A ; Y)-\delta_{Y} \alpha(A ; X)-\alpha(A ;[X, Y]) \\
& =\frac{i}{12 \pi^{2}} \int \operatorname{tr} d A(d X d Y+d Y d X) .
\end{aligned}
$$

Since the form under the integral sign is the exterior derivative of the three-form $\operatorname{tr} A[d X, d Y]_{+}$, the two-cocycle $c_{3}$ defines in fact an extension of the current algebra $\operatorname{Map}\left(S^{3}, \mathrm{~g}\right)$ by the abelian ideal $\operatorname{Map}\left(\mathscr{A}_{3}, i \mathbb{R}\right)$,

$$
[(X, \lambda),(Y, \mu)]=\left([X, Y], \delta_{X} \mu-\delta_{Y} \dot{\lambda}+i c_{3}(A ; X, Y)\right)
$$

with

$$
c_{3}(A ; X, Y)=\frac{i}{12 \pi^{2}} \int_{S^{3}} \operatorname{tr} A(d X d Y+d Y d X)
$$

Because of the "Schwinger term" $c_{3}$ in the commutation relations, one cannot impose the Gauss' law constraint in the space of Schrödinger wave functions $\psi(A)$ : The integrals

$$
\int Q^{i}(\mathbf{x}) f(\mathbf{x}) d^{3} x
$$

of the charge densities $Q^{i}(\mathbf{x})$ [ $i$ is a $S U(N)$ Lie algebra index] are represented in the Hamiltonian formulation by operators satisfying the commutation relations (30); setting $Q^{i} \equiv 0$ leads to inconsistencies with (30).

Let us finally consider the case $G=S U(2)$. From the elementary properties of trace follows that now $\omega(f, g) \equiv 0$. However, we can still define a non-trivial $U(1)$ bundle on $\Omega_{3} G$. Namely, we can define a relation " $\sim$ " in $D_{4} G \times U(1)$ by

$$
(f, \lambda) \sim(f g, \lambda \varepsilon(g))
$$

where again $g \in \mathscr{G}_{4}$ and $\varepsilon(g)=+1$ if $g: S^{4} \rightarrow S U(2)$ is contractible and $\varepsilon(g)=-1$ otherwise [note that $\pi_{4} S U(2)=\mathbb{Z}_{2}$ ]. Now $D_{4} G \times U(1) / \sim$ is a principal $U(1)$-bundle such that the structure group of the bundle reduces to $\mathbb{Z}_{2}$ by construction. Let us denote by $P_{3}^{(1)}$ this bundle and let $P_{3}^{(0)}$ be the trivial $U(1)$-bundle on $\Omega_{3} G$.

There is a natural flat connection in $P_{3}^{(1)}$ such that the parallel transport around a non-contractible closed loop in $\Omega_{3}$ gives the group element -1 in $\mathbb{Z}_{2}$. The horizontal space of the connection is represented in $D_{4} G \times U(1)$ by vectors which are tangential to $D_{4} G$. From this definition we can see that the parallel transport is given as follows. Let $t \mapsto f_{t}$ be a path in $\Omega_{3} G(0 \leqq t \leqq 1)$. Take a lift $t \rightarrow \widetilde{f}_{t} \in D_{4} G$, $\left.\widetilde{f}_{t}\right|_{S^{3}}=f_{t}$. Then the parallel transport of $\left[\left(\tilde{f}_{0}, \lambda_{0}\right)\right]$ along the path $f_{t}$ gives by definition the element $\left[\left(\tilde{f}_{1}, \lambda_{0}\right)\right]$ at $f_{1}$. The parallel transport does not depend on the choice of the lift $f_{t}$. If $t \rightarrow h_{t} \in D_{4} G$, such that $\left.h_{t}\right|_{S^{3}}=f_{t}$ and $h_{0}=\widetilde{f}_{0}$, then $h_{1}=\widetilde{f}_{1} g$ for some $g \in \mathscr{G}_{4}$. How $g_{t}=\widetilde{f}_{t}^{-1} h_{t}$ is a contraction of $g=g_{1}$ to $g_{0}=1$ and therefore $\varepsilon(g)=1$. Thus $\left(h_{1}, \lambda_{0}\right) \sim\left(\widetilde{f}_{1}, \lambda_{0}\right)$. If we have a closed loop $f_{0}=f_{1}$, then $\widetilde{f}_{1}=\widetilde{f}_{0} g$ for some $g \in \mathscr{G}_{4}$ depending on the loop $f_{t}$; it is clear that $g$ represents the non-trivial element of $\pi_{4} S U(2)$ if and only if $f_{t}$ is non-contractible and it follows that the parallel transport around $f_{t}$ is given by the group element +1 for contractible $t \mapsto f_{t}$ and by -1 for a non-contractible loop.

Corresponding to $P_{3}^{(k)}$ we have two different choices for the determinant bundle $\operatorname{Det}_{4}^{(k)}, k=1,2$. The bundle $\operatorname{Det}_{4}^{(0)}$ is trivial but $\operatorname{Det}_{4}^{(1)}$ has a non-trivial $\mathbb{Z}_{2}$ structure; the situation is the same for the associated line bundles $E_{3}^{(k)}$ to $P_{3}^{(k)}$. [The existence of two types of bundles follows from the fact that $\pi_{2}\left(\Omega_{3} S U(2)\right)=\pi_{5} S^{3}=\mathbb{Z}_{2}$; for $N \geqq 3$ 
we have $\pi_{5} S U(N)=\mathbb{Z}$ and therefore for each $k \in \mathbb{Z}$ we can construct a nonequivalent bundle defined by the cocycles $\omega=\omega_{0}+k C_{5}$.]

The considerations above can be extended to all sectors $\Omega_{3}^{(n)} G$ of the base space ( $n \in \mathbb{Z}$ is the soliton number). A typical $n$-soliton is the map $x \mapsto x^{n}$ from $S^{3}=S U(2)$ to $S U(2)$. If $g \in \Omega_{3}^{(n)}$, then $f(x)=x^{-n} g(x)$ is an element of $\Omega_{3}^{(0)}$ and the map $g \mapsto f$ is a homeomorphism $\Omega_{3}^{(n)} \rightarrow \Omega_{3}^{(0)} \quad$ [we use the natural metric topology in $\operatorname{Map}\left(S^{3}, S U(N)\right)$ defined by the Killing metric of $\left.S U(N)\right]$. Thus the topological structure of the bundle $P_{3}$ can be studied by restricting the base space $\Omega_{3}$ to the connected component $\Omega_{3}^{(0)}$ (as was done above).

Consider the space $\Gamma\left(E_{3}^{(k)}\right)$ of sections of $E_{3}^{(k)}$. We want to show that a rotation by the angle $2 \pi$ in the compactification $S^{3}$ of the physical space $\mathbb{R}^{3}$ induces a multiplication by \pm 1 on the sections. First, we can write

$$
\Gamma\left(E_{3}^{(k)}\right)=\Gamma_{+} \oplus \Gamma_{-},
$$

where $\Gamma_{+}$(respectively $\Gamma_{-}$) is the space of sections with support in $\bigcup \Omega_{3}^{(n)} G$, where $n$ takes all even (respectively odd) integral values. A typical element of $\Omega_{3}^{(n)} G$ is the $n$ soliton

$$
f(\mathbf{x})=\exp i h(r) \frac{\mathbf{x}}{r} \cdot \boldsymbol{\sigma}
$$

where $h:[0, \infty] \rightarrow \mathbb{R}$ is monotonously increasing with $h(0)=0$ and $h(\infty)=n \cdot 2 \pi ; \sigma$ is the set of Pauli matrices. Let

$$
f_{t}(\mathbf{x}):=e^{i \pi t \sigma_{3}} f(x) e^{-i \pi t \sigma_{3}},
$$

where $0 \leqq t \leqq 1$ is interpreted as the time coordinate. As $t$ goes from 0 to 1 , the soliton is rotated once around the third coordinate axes. As explained by Witten [7], the path $t \mapsto f_{t}$ is non-contractible (represents the element -1 in $\pi_{4} G$ ) iff $n$ is odd; the parallel transport in $E_{3}^{(1)}$ around $f_{t}$ is a multiplication by $(-1)^{n}$. The same result holds for any map $g: S^{3} \rightarrow G$ which is homotopic to $f$, and so the parallel transport around any line of flow in $\Omega_{3}^{(n)}$ induced by the rotations exp $i \pi t \sigma_{3}$ gives a factor $(-1)^{n}$ to the sections which have support in $\Omega_{3}^{(n)}$. Thus the rotations by the angle $2 \pi$ are represented by +1 in $\Gamma_{+}$and by -1 in $\Gamma_{-}$.

The determinant line bundle $\operatorname{Det}_{4}^{(1)}$ is the pull-back of $E_{3}^{(1)}$ with respect to the homotopy equivalence $\mathscr{A}_{4} / \mathscr{G}_{4} \rightarrow \Omega_{3} G$, and it follows that what has been said above holds also in the space $\Gamma\left(\operatorname{Det}_{4}^{(1)}\right)$ of sections of $\operatorname{Det}_{4}^{(1)}$.

We come now to our main conclusion concerning the case $G=S U(2)$, which is a generalization of Witten's fermionization mechanism: The apparently bosonic Schrödinger wave functions $\psi$ (= sections of the determinant bundle $\left.\operatorname{Det}_{4}^{(1)}\right)$ can describe both bosons and fermions, depending only on the topological nature of the support $\operatorname{supp} \psi$ of $\psi$. If supp $\psi$ belongs to the odd instanton number sector in $\mathscr{A}_{4} / \mathscr{G}_{4}$, then $\psi$ is a fermion; if supp $\psi$ is contained in the even instanton number sector, then $\gamma$ is a boson. Of course, sections of the topologically trivial bundle $\operatorname{Det}_{4}^{(0)}$ can be only bosons.

The geometry and the topology of the determinant line bundle has recently been discussed from different points of view in several papers. For example, Nelson and Alvarez-Gaumé [12] have determined the $\mathbb{Z}_{2}$ structure of the bundle [when $G=S U(2)]$ using index theory arguments, and they have clarified also the typical implications (pair production). The space $\mathscr{A}_{2 n} / \mathscr{G}_{2 n}$ has a presymplectic structure, which has been studied in [6] and in more detail by Bao and Nair [13]. The presymplectic structure is directly related to the Schwinger terms in current 
algebra commutation relations. For more information on Schwinger terms in addition to [4], see refs. [14].

The idea to use a non-trivial connection in the configuration space for "fermionization" was used earlier in [15] in classical mechanics.

\section{References}

1. Segal, G., Wilson, G.: Loop groups and equations of KdV type. IHES Publ. Math. 61, 5 (1985)

2. Ueno, K., Nakamura, Y.: Transformation theory for anti-self-dual equations. Publ. Res. Inst. Math. Sci. (Kyoto) 19, 519 (1983)

3. Gross, D.J., Harvey, J., Martinec, E., Rohm, R.: Heterotic string theory (I). The free heterotic string. Nucl. Phys. B 256, 253 (1985)

4. Mickelsson, J.: On a relation between massive Yang-Mills theories and dual string models. Lett. Math. Phys. 7, 45 (1983); Chiral anomalies in even and odd dimensions. Commun. Math. Phys. 97, 361 (1985); Bose-Fermi correspondence and Kac-Moody algebras in four dimensions. Phys. Rev. D 32, 436 (1985)

5. Pressley, A.N., Segal, G.: Loop groups and their representations. Oxford: Oxford University Press 1986

6. Mickelsson, J.: Two-cycle of a Kac-Moody group. Phys. Rev. Lett. 55, 2099 (1985)

7. Witten, E.: Current algebra, baryons, and quark confinement. Nucl. Phys. B 223, 433 (1983)

8. Finkelstein, D., Rubinstein, J.: Connection between spin, statistics, and kinks. J. Math. Phys. 9, 1762 (1968)

9. Atiyah, M., Singer, I.: Dirac operators coupled to vector potentials. Proc. Natl. Acad. Sci. (USA) 81, 2597 (1984)

Alvarez:Gaumé, L., Ginsparg, P.: The topological meaning of non-abelian anomalies. Nucl. Phys. B 243, 449 (1984)

10. Singer, I.: Some remarks on the Gribov ambiguity. Commun. Math. Phys. 60, 7 (1978); Physica Scripta 24, 817 (1981)

11. Goodman, R., Wallach, N.: Structure and unitary cocycle representations of loop groups and the diffeomorphisms of the circle. J. Math. 347, 69 (1985)

12. Nelson, P., Alvarez-Gaumé, L.: Hamiltonian interpretation of anomalies. Commun. Math. Phys. 99, 103 (1985)

13. Bao, D., Nair, V.P.: A note on the covariant anomaly as an equivariant momentum mapping. Commun. Math. Phys. 101, 437 (1985)

14. Jackiw, R., Johnson, K.: Anomalies of the axial-vector current. Phys. Rev. 182, 1459 (1969) Jo, S. Commutator of gauge generators in non-abelian chiral theory. Nucl. Phys. B 259, 616 (1985)

Faddeev, L.: Operator anomaly for the Gauss law. Phys. Lett. 145 B, 81 (1984);

Niemi, A., Semenoff, G.: Quantum holonomy and the chiral gauge anomaly. Phys. Rev. Lett. 55, 927 (1985)

Zumino, B.: Berkeley preprints (1985)

Segal, G.: Oxford preprint (1985)

15. Mickelsson, J.: Geometry of spin and statistics in classical and quantum mechanics. Phys. Rev. D 30, 1843 (1984)

16. Witten, E.: Non-abelian bosonization in two dimensions. Commun. Math. Phys. 92, 455 (1984)

17. Zumino, B.: Chiral anomalies and differential geometry. In: Les Houches Proc. 1983, de Witt, B., Stora, R. (eds.). Amsterdam: North-Holland

18. Bardeen, W.: Anomalous Ward identities in spinor field theories. Phys. Rev. 184, 1848 (1969)

Communicated by S.-T. Yau

Received February 17, 1986; in revised form July 8, 1986 
